•综述・

\title{
结构稳定性：概念、方法和应用
}

\author{
宋础良 ${ }^{1,2,3 *}$ \\ 1 (Department of Civil and Environmental Engineering, Massachusetts Institute of Technology, Cambridge, MA 02139, USA) \\ 2 (Department of Biology, McGill University, Montreal, H3A 1B1, Canada) \\ 3 (Department of Ecology and Evolutionary Biology, University of Toronto, Toronto, Ontario M5S 3B2, Canada)
}

\begin{abstract}
摘要: 群落内物种间相互作用的结构是高度组织化的。群落结构对多物种共存的影响机制是群落生态学的核心科 学问题之一。目前生态学界在这一问题上存在多种不同的观点。一个可能的原因是, 由于环境因子的复杂性, 大 部分研究忽略了环境因子对群落结构和物种共存的重要影响。在这一背景下, 近期发展起来的结构稳定性理论系 统地联系了群落结构、环境因子和物种共存, 并在此基础上建立了一个和经验数据紧密结合的理论框架。本文首 先简要回顾了当前关于群落结构研究的争鸣, 然后介绍了结构稳定性的理论框架和计算方法, 最后详细介绍了结 构稳定性理论在不同生态群落和不同生态学问题中的应用。在全球气候变化的背景下, 结构稳定性理论提供了一 种新的视角来理解群落层面的生物多样性维持机制。
\end{abstract}

关键词: 群落结构; 物种共存; 结构稳定性; 环境梯度; 环境胁迫

\section{Structural stability: Concepts, methods, and applications}

Chuliang Song ${ }^{1,2,3^{*}}$

1 Department of Civil and Environmental Engineering, Massachusetts Institute of Technology, Cambridge, MA 02139, USA

2 Department of Biology, McGill University, Montreal, H3A 1B1, Canada

3 Department of Ecology and Evolutionary Biology, University of Toronto, Toronto, Ontario M5S 3B2, Canada

\begin{abstract}
Ecological networks-how species interactions are organized within ecological communities—are highly structured, which has motivated generations of ecologists to elucidate how these structures affect species coexistence. Unfortunately, we still do not have a clear and consistent answer about the link between network structure and species coexistence. A possible explanation is that most of the studies do not take into account that the environment affects both network structure and species coexistence due to the multidimensional and changing nature of environmental factors. In this context, the structural stability approach provides a theoretical framework grounded on biological realism to quantitatively link network structure, species coexistence, and environmental factors. I begin by an overview of the heated debates in the study of ecological networks. Then I introduce the theoretical framework and computational tools of the structural stability approach in a nutshell. Then I show the empirical applications in different ecological questions across a broad range of ecological systems. Overall, the structural stability approach provides a new perspective to understand the maintenance of biodiversity in ecological communities.
\end{abstract}

Key words: community structure; species coexistence; structural stability; environmental gradient; environmental stress

没有一个物种是一个孤岛(Lawton, 1999)。地求 上几乎每一种生命的存活都在很大程度上依赖于 与其他物种的相互作用。群落内多物种共存的基本
原理是群落生态学的核心问题之一(Hutchinson, 1978; Levine et al, 2017)。生态学家提出了许多理论 框架来解释物种为什么可以共存(综述可见Vellend, 
2016)。其中的代表性理论包括现代生态位理论 (Tilman, 1982; Chase \& Leibold 2003; 李德志等, 2006)、现代共存理论(Chesson, 2000; Adler et al, 2007; 储诚进等, 2017)、中性理论(Hubbell, 1997; 周淑荣和张大勇, 2006; Zhou \& Zhang, 2008)和最大 熵理论等(Harte, 2011; 邢丁亮和郝占庆, 2011; Harte \& Newman, 2014)。然而, 这些经典理论往往 难以直接应用于自然群落, 并解释其中的物种共存 (Levine et al, 2017)。困难的核心在于如何处理群落 尺度上环境因子的不确定性(Levin, 1992; Lawton, 1999; Wang, 2018)。简单来说, 自然界中的群落往往 介于两个极端尺度之间：(1)一两个物种的小尺度, 实验往往可以有效控制环境因子的不确定性，继而 揭示出因果机制; (2)上百乃至上千个物种的大尺度, 不确定的环境因子不影响统计规律的涌现 (emergence)。例如, 现代生态位理论和现代共存理 论更适用于小尺度(Letten et al, 2017; Barabás et al, 2018; Song et al, 2019a), 而中性理论和最大熵理论 更适用于大尺度(Azaele et al, 2016; O’Dwyer et al, 2017)。然而, 很多群落属于中间尺度(mesocale)。

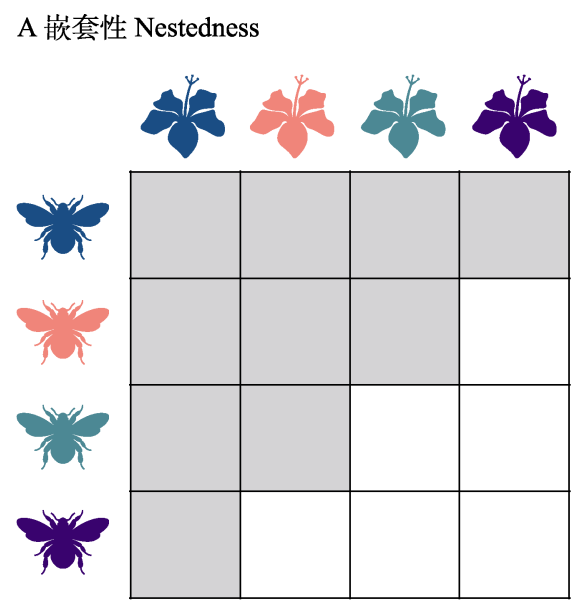

很多群落生态学家认为在群落尺度上可能不存在 普适性的物种共存机制 (Lawton, 1999; Currie, 2019)。

面对群落尺度的复杂性, 群落生态学兴起的一 种新范式是研究群落结构(community structure)。群 落结构指的是群落内物种之间相互作用的网络结 构。具体而言, 将每一个物种视为节点, 物种间的 相互作用视为边, 那么一个群落就对应于一个网络 (May, 2006)。研究群落结构的核心目标是寻找不同 群落中的普适结构特征(universality), 从而揭示不 同群落中通用的物种共存机制(Garlaschelli et al, 2003; Proulx et al, 2005; Bascompte, 2010)。普适结 构特征指的是不同的生态群落所共有的群落结构 特征。例如, 很多群落生态学家认为, 模块化 (modularity) 是食物网中的一种普适结构特征 (Thébault \& Fontaine, 2010; Stouffer \& Bascompte, 2011; Sales-Pardo, 2017), 而嵌套性(nestedness)是互 利群落中的一种普适结构特征(Bascompte et al, 2003; Mariani et al, 2019)。模块度和嵌套性的图示 参见图1。

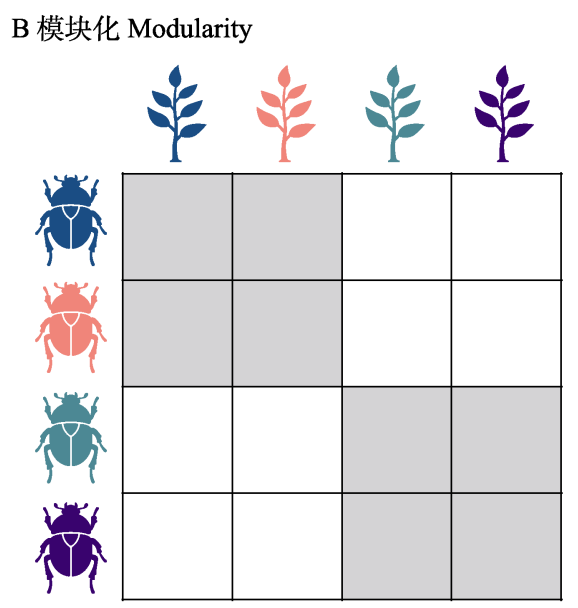

图1 两种经典的群落结构。这里的矩阵展示了群落结构: 每一列代表不同植物, 而每一行代表了不同的授粉种(图A)或者草 食动物(图B)。灰色的格子代表两个物种存在种间关系, 而白色的格子则代表不存在。图A展示了嵌套性结构。基于一个物种 的种间关系的相对数量, 可以把物种分为泛化种(generalist)和特化种(specialist)。嵌套性结构的特征是认为泛化种会和泛化 种以及特化种都相互作用, 而特化种几乎只和泛化种相互作用。嵌套性结构被认为是互利群落的一种普适结构。图B展示了 模块化结构。模块化结构的特征是群落分成几个模块, 物种几乎只和同一模块的物种相互作用, 而很少和别的模块的物种相 互作用。嵌套性结构被认为是食物网的一种普适结构。

Fig. 1 Two ecological community structures. Here we present an illustration of two hypothetical network structures. The community structure is represented as a matrix. The columns correspond to different plant species while the rows correspond to different pollinators (in Panel A) or herbivores (in Panel B). A species interaction is represented as a gray grid. Panel (A) illustrates the nested structure. The defining feature of a nested structure is that highly connected species interact with both highly connected and poorly connected species, while poorly connected species interact almost exclusively with highly connected species. The nested structure is widely conceived as a universal structure in mutualistic communities. Panel (B) illustrates the modular structure. The defining feature of the modular structure is that in which groups of species have many interactions among them, but few interactions with the rest of the species in the network. The modular structure is widely conceived as a universal structure in food webs. 
然而, 这一追寻群落结构普适性的范式在近十 年受到了很多的挑战。其中最大的一个挑战是很多 观测到的群落结构并不符合所谓的普适结构 (Michalska-Smith \& Allesina, 2019)。最初, 很多群落 生态学家将这些观测结果和普适结构之间的差异 视为噪声。然而, 伴随着越来越多的实证数据, 这 些差异已经远远不是简单的噪声可以解释的。一个 新兴的研究思路是去找寻每一个群落所独有的特 异性结构特征(particularity)。特异性结构特征指的 是一个生态群落的群落结构与普适结构特征的区 别。如前所述, 群落受到环境因子的影响, 所以不 能简单地期望所有群落都有完全相似的结构特征。 恰恰相反, 特异性结构隐藏着群落所对应的环境因 子的重要线索。通过研究环境因子和特异性结构的 关系, 可以更好地理解普适性结构, 进而揭示普适 的物种共存机制。

基于这一新的研究思路, 结构稳定性(structural stability)理论尝试将群落结构和环境因子系统地联 系在一起来解释物种共存。粗略而言, 结构稳定性 定量地刻画了在给定的群落结构下允许物种共存 的所有环境条件。具体而言, 当给定一个群落结构, 如果物种只有在很特定的环境条件下才能共存, 那 么这个群落的结构稳定性很低; 反过来, 如果物种 在很普遍的环境条件下都可以共存, 那么这个群落 的结构稳定性很高。因此, 直观上结构稳定性可以 理解成物种共存的概率: 如果一个群落具有更高的 结构稳定性, 那么在环境变化下这个群落中的物种 有更大概率可以继续共存(Song et al, 2018b)。通过 结构稳定性这一工具, 研究者系统地构建了基于环 境因子和群落结构的物种共存的理论。

结构稳定性最初是由研究动力系统的数学家 提出的数学概念(Bažant, 2000)。数学家René Thom 的奠基性工作将结构稳定性引入了生物的形态发 生学(Morphogenesis)研究中(Thom, 1972)。在理论群 落生态学的发展初期, 理论生态学家就已经意识到 结构稳定性的重要性(Lewontin, 1969; May, 1975)。 遗憾的是, 结构稳定性并没有引起广泛的重视, 只 有少数理论生态学家在一些生态学模型中对其有 零星的讨论(Jansen \& Sigmund, 1998; Meszéna et al, 2006; Rossberg, 2013)。由Rudolf P. Rohr和Serguei Saavedra对其重新刻画之后(Rohr et al, 2014), 结构 稳定性才在群落生态学中得到较为广泛的关注。尽
管只有短短几年的发展，结构稳定性理论在物种共 存的研究领域已成为一股重要的新生力量。

本文首先简要回顾当前群落结构的研究争议, 在此基础上解释引入结构稳定性的动机。然后将详 细介绍结构稳定性的基础数学框架及其拓展。再重 点介绍结构稳定性理论所构建的物种共存的理论 框架，以及支撑这一理论框架的实证证据。最后对 结构稳定性未来的研究方向进行展望。

\section{群落结构的争鸣和结构稳定性的动机}

\section{1 理论争鸣: 以嵌套性结构为例}

互利(mutualism)是自然界中最为常见的种间关 系之一。自21世纪初发现嵌套性结构开始(Bascompte et al, 2003), 互利群落的嵌套性结构是过去 15 年来群落生态学界的热点话题之一, 仅仅在 Nature和 Science上就有多篇研究论文(Bastolla et al, 2005; Montoya et al, 2006; Thébault \& Fontaine, 2010; Saavedra et al, 2011; Allesina \& Tang, 2012; James et al, 2012; Suweis et al, 2013; Rohr et al, 2014; Guimarães et al, 2017), 在专业生态学期刊上的论文 数量更为可观(综述可见Mariani et al, 2019)。

虽然开展了大量研究, 但生态学家并未在嵌套 性结构上达成共识。概括而言，研究者分成了三个 派系。第一个派系认为嵌套性结构是支撑互利群落 的物种多样性的核心因素(Bastolla et al, 2009; Suweis et al, 2013; Rohr et al, 2014); 第二个派系认为 嵌套性结构和物种多样性之间没有因果关系(James et al, 2012, 2013; Barabás et al, 2016; Fort et al, 2016; Valverde et al, 2018); 第三个派系认为嵌套性结构 在自然界的互利群落中并不是普适性的结构特征 (Staniczenko et al, 2013; Michalska-Smith \& Allesina, 2019; Payrató-Borràs et al, 2019)。图2显示了不同派 系在嵌套性结构和物种共存之间的因果关系上所 秉持的观点差异。

显然，这三种派系的观点不可能同时正确。类 似的争议也同样存在于对其他类型的生态群落(例 如食物网)的研究中(May, 1972; Solow et al, 1999; Stouffer \& Bascompte, 2011; Grilli et al, 2016; Cenci et al, 2018b)。这些派系间的争论涉及到群落结构的 本质, 所以怎样消解这些争论是当前群落结构研究 的一个核心问题。 


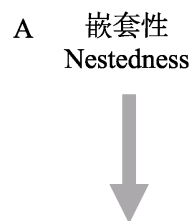

物种共存

Species coexistence
B

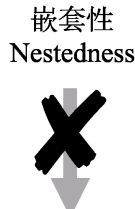

物种共存 Species coexistence
C

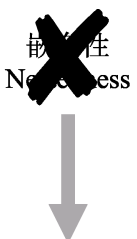

物种共存 Species coexistence
图2 群落结构研究的争鸣。互利群落中关于嵌套性和物种 多样性的研究中, 存在三种不同的派系。图A展示了第一种 派系, 认为嵌套性是决定互利群落的物种多样性的原因。图 B展示了第二种派系, 认为嵌套性并不是决定互利群落的物 种多样性的原因。图C展示了第三种派系, 认为嵌套性并不 是互利群落的普适结构, 因此嵌套性和物种多样性之间的 关系无从谈起。

Fig. 2 The debates in the study of community structures. Three different schools of thought coexist in linking nestedness and species coexistence in mutualistic communities. The first school (Panel A) argues that nestedness is a key factor to support the biodiversity in mutualistic communities, the second school (Panel B) argues there is no causal relationship between the nested pattern and biodiversity, and the third school (Panel C) argues that nested patterns are not universal in observed mutualistic communities, thus makes the whole question a straw man.

\section{2 引入结构稳定性的动机}

这三个派系都忽略了环境因子的重要性。环境 因子不仅会影响物种共存(Scheffers et al, 2016; Song et al, 2020b), 而且会影响群落结构(Song et al, 2017; Tylianakis \& Morris, 2017; Pellissier et al, 2018)。图3展示了物种共存、群落结构和环境因子 之间的因果关系。用因果推断的术语来说, 环境因 子这类同时影响原因(群落结构)和结果(物种共存) 的因素被称为混杂因子(confounder) (Pearl, 2009)。 因果推断的理论表明, 如果不控制混杂因子, 很容 易会得到错误的因果关系(Pearl, 2009)。所以, 派系 间争鸣的起源很可能是对环境因子的忽视。

结构稳定性正是把环境因子和群落结构结合在 一起来研究物种共存。物种生存所处的环境一直在 变化, 所以一个可持续的群落中的物种必须要在不 同的环境下共存。结构稳定性研究的是给定的群落 结构下物种共存所可以容忍的全部环境条件。结构 稳定性理论的核心观点是把环境因子的不确定性转 化为群落动力学中参数的不确定性: 如果环境因子 的不确定性更大, 那么物种必须能够在更大的参数 空间(parameter space)下共存(Song et al, 2018b)。因 此, 通过结构稳定性这一工具, 群落结构和物种共

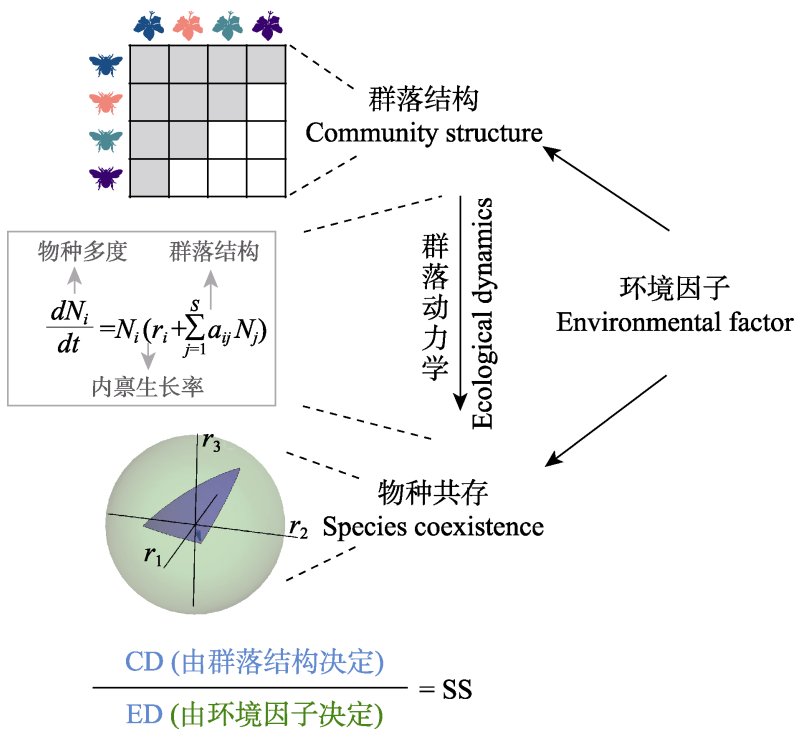

图3 结构稳定性理论的基础数学框架。和经典的群落结构物种共存理论相比, 结构稳定性理论引入了环境因子。环境 因子同时会影响群落结构和物种共存。结构稳定性(SS)的计 算需要两部分的信息: 共存域(CD)和环境域(ED)。共存域指 的是可以使得物种共存的全部参数的空间, 图中用蓝色区 域(图A)指代。共存域是由群落结构通过群落动力学而决定 的。环境域指的是这一环境条件下所有可能的参数的空间, 图中用绿色区域(图B)指代。环境域是在给定环境的限制下 所有可能的参数的空间。环境域是环境因子决定的。结构稳 定性是共存域相对于环境域的大小。结构稳定性越大, 物种 在这一环境条件下就有更大可能可以共存。

Fig. 3 The mathematical framework of the structural stability approach. To compute the structural stability (SS) of an ecological community, we need two pieces of information: the coexistence domain (CD) and the environment domain (ED). The coexistence domain (denoted in blue; region A) is the full range of parameters that are compatible with species coexistence. The coexistence domain is determined by the community structure via the ecological dynamics. The environment domain (denoted in green; region B) is the full range of parameters constrained by the given environmentally conditions. The environment domain is determined by the environmental factors. Structural stability is defined as the relative size of the coexistence domain comparing to the environment domain. The larger the structural stability is, the more likely species can coexist under the given environmental conditions.

存的研究可以有效地考虑环境因子的作用。

\section{结构稳定性的数学框架}

\section{1 多物种的种群动力学模型和可行性}

对于一个包含 $S$ 个物种的群落, 描述这个群落 内多物种相互作用的最基本的动力学模型是 Lotka-Volterra模型: 


$$
\frac{1}{N_{i}} \frac{d N_{i}}{d t}=r_{i}+\sum_{j=1}^{S} a_{i j} N_{j}
$$

其中 $N_{i}$ 是物种 $i$ 的多度, $r_{i}$ 是物种 $i$ 的内禀增长率, $a_{i j}$ 是 物种 $j$ 对物种 $i$ 的作用强度。需要注意的是, 这里种内 和种间的相互作用 $\left\{a_{i j}\right\}$ 指代的就是群落结构。

在群落的平衡态 $N_{i}^{*}$, 物种多度不发生变化 $\left(\frac{d N_{i}}{d t}=0\right)$ 。代入动力学模型(公式1), 我们得到

$$
r_{i}+\sum_{j=1}^{S} a_{i j} N_{j}^{*}=0
$$

可行性(feasibility)指的是在群落的平衡态 $N_{i}^{*}$ 时物种的多度都是正值(Roberts, 1974)。换言之, $N_{i}^{*}>0, \forall i$ 。可行性是物种共存的必要条件, 即使群 落处于非平衡态时也需要满足可行性(Hofbauer \& Sigmund, 1998)。对于所有衡量物种共存的条件, 例 如动态稳定性 (dynamical stability)、可入侵性 (invasibility)和持续性(permanence)等, 都必须同时 满足可行性(Song \& Saavedra, 2018b)。

\section{2 结构稳定性的定义和定量计算}

如前所述, 结构稳定性研究的是一个群落结构 所决定的物种共存可以容忍的全部环境条件。这里 我们讨论如何在数学上来定义这一概念。

我们在此做出两个假设(这两个假设是可以放 松的, 详述见后)。第一个假设认为内禀增长率反映 了环境条件, 因为内禀增长率很大程度上决定于环 境条件(Levins, 1968; Roughgarden, 1975; Meszéna et al, 2006; Coulson et al, 2017)。第二个假设将可行 性视为物种共存的定义。基于这两个假设, 我们可 以定义共存域和环境域: 共存域指的是在一个给定 的群落结构下 $\left(\left\{a_{i j}\right\}\right)$ 所有满足可行性的内禀增长率 的集合; 而环境域指的是在给定的环境因子下所有 可能的内禀增长率的集合。共存域取决于群落结构 特征, 而环境域则取决于环境因子。那么, 结构稳 定性的定义就是在环境域内落在共存域的条件概 率。换言之, 就是落在共存域和环境域的交集的可 能性相对于落在环境域的可能性。

结构稳定性 $=\mathbb{P}$ (共存域 $\mid$ 环境域 $)=$

$$
\frac{\mathbb{P}(\text { 共存域环境域 })}{\mathbb{P} \text { (环境域 })}
$$

结构稳定性的这一数学定义在生态学上可以
理解为物种在给定的群落结构和可能的环境范围 下共存的概率。图3展示了这个定义。

如果进一步假设所有的环境条件都具有相同 的可能性 (存在概率), 则结构稳定性的解析解为 (Ribando, 2006; Saavedra et al, 2016b):

$$
\begin{gathered}
\text { 结构稳定性 }=\frac{1}{(2 \pi)^{S / 2} \sqrt{|\operatorname{det}(\boldsymbol{A})|}} \\
\int \cdots \int_{N_{i}^{*} \geqslant 0} \exp \left(-\frac{1}{2} N^{* T} \boldsymbol{A}^{T} \boldsymbol{A} N^{*}\right) d N^{*}
\end{gathered}
$$

即使对于物种丰富度很高的群落, 拟 Monte-Carlo方法也可以用于有效地计算结构稳定 性的数值解(Genz \& Bretz, 2002; Grilli et al, 2017; Song et al, 2018b)。

\section{3 结构稳定性和其他物种共存概念的差别}

结构稳定性和其他描述物种共存的概念有本 质区别。概括而言, 其他描述物种共存的概念可以 分为两类。

第一类概念描述的是固定全部参数后群落的 动力学性质。这一类概念有可行性、动态稳定性、 可入侵性和持续性等。这一类的动力学性质研究的 都是在给定的参数下是否成立。例如, 可行性指的 是在所有的参数 (内禀增长率和群落结构) 都固定的 情况下, 群落的平衡态的物种多度是否是正的 (Case, 2000)。然而, 结构稳定性研究的是这一类动 力学性质在哪些参数范围下成立, 所以在概念的层 次上是不同的。例如, 我们之前讨论的是可行性的 结构稳定性, 但我们也同样可以考虑动态稳定性的 结构稳定性或者持续性的结构稳定性等等(Song et al, 2020b)。

第二类概念是敏感度分析(sensitivity analysis)。 敏感度分析描述的是对参数的微小扰动下某些生 态学变量(例如多度)的变化(Rossberg, 2013; Barabás et al, 2014a, b)。敏感度分析和结构稳定性的本 质区别是敏感度分析只能考虑参数的微小扰动, 而 结构稳定性则考虑全部的参数范围。

需要注意的是, 以上的辨析只是为了避免概念 上的混淆, 而绝非说结构稳定性比其他描述物种共 存的概念更好。以上每一个概念都只是对物种共存 这一宏大现象的盲人摸象, 因而都有着其适用范围。

\section{4 拓展的理论框架}

以上介绍的只是结构稳定性最基本的数学框 
架。不难看出, 这一基本的数学框架中存在多种假 设。后续的理论工作在不同程度上放松了这些假设, 使得结构稳定性可以运用于更真实的群落模型。本 文简要描述以下三方面的主要进展:

(1)拓展到更广泛的种群动力学模型。我们之前 使用了Lotka-Volterra模型(公式1)来描述群落动力 学。Lotka-Volterra模型对种间相互作用有两个核心 的假设。第一个假设是种间相互作用都是线性的功 能响应(functional response), 但线性的功能响应往 往是不现实的(Bascompte et al, 2006; Holland et al, 2006)。多项理论工作修正了这一假设, 将基本的结 构稳定性理论推广到了许多具有非线性功能响应 的种群动力学模型(Pascual-García \& Bastolla, 2017; Butler \& O’Dwyer, 2018; Cenci \& Saavedra, 2018; Dougoud et al, 2018)。第二个假设是种间相互作用 都是两两间的(pairwise interaction), 而没有考虑高 阶相互作用(higher-order interaction) (Levine et al, 2017)。新的理论工作放松了这一假设, 讨论了引入 高阶物种相互作用对结构稳定性的影响(AlAdwani \& Saavedra, 2019; Letten \& Stouffer, 2019)。

(2)引入环境条件的概率分布。我们之前假设环 境条件的概率为均匀分布(所有环境条件具有相同 的概率)。这显然是一个过于简化的假设。后续研究 对这一假设做了两部分的修正。第一种修正是引入 营养级约束(trophical constraints)。具体地, 假定处 于第一营养级的物种的内禀增长率是正的, 而处于 更高营养级的物种的内禀增长率则是负的。通过引 入营养级约束, 我们可以缩小可能的环境条件, 从 而得到更精确的结构稳定性的估计 (Song et al, 2018b)。第二种修正是通过贝叶斯分析得到环境条 件更为准确的概率分布。很多实证工作可以提供环 境条件的后验概率分布(Uricchio et al, 2019)。通过 在公式(3)中加入不同环境条件的权重, 就可以更准 确地估计结构稳定性。

(3)拓展结构稳定性的定义。我们之前只考虑了 环境因子对内禀增长率的影响, 但环境因子显然会 影响群落的多个方面。后续研究对这一假设做了两 部分的修正。第一个修正是定义了关于群落结构的 结构稳定性。环境因子会改变群落结构, 包括种间 的相互作用关系(Callaway et al, 2002)以及作用的强 度(Ushio et al, 2018)。之前我们通过固定群落结构
来定义了关于内禀增长率的结构稳定性, 类似地, 可以固定内禀增长率来定义关于群落结构的结构 稳定性(Chesson, 2018; Song et al, 2020a)。第二个修 正是估计群落的局部结构稳定性。群落的局部结构 稳定性指的是动力学系统中的局部的雅各布矩阵 (Jacobian matrix)的迹(即矩阵对角线元素的总和) (Strogatz，2014)。群落的局部结构稳定性的重要性 质是: 如果群落局部的结构稳定性越大, 那么相对 于同样的环境扰动, 群落局部的多度变化也会越小, 反之亦然。和之前定义的全局结构稳定性不同，局 部结构稳定性会随着时间而变化。通过使用非参数 统计学方法(Sugihara, 1994; Deyle et al, 2016b; Cenci et al, 2019; Song \& Saavedra, 2020a), 可以从 种群多度的时间序列中直接估计群落在任一时刻 的局部结构稳定性(Cenci \& Saavedra, 2019)。

\section{3 基于结构稳定性的物种共存理论和实证请}

结构稳定性试图建立一套系统的群落层面的 物种共存理论。图4展示了生态群落的核心要素(物 种库、群落结构、环境因子和物种共存)以及它们之 间的影响关系。下面将具体阐述如何建立和量化这 些变量的关系及其实证证据。

\section{1 群落结构如何影响物种共存}

群落动力学在群落结构和物种共存之间建立 了联系(May, 2006; Levine et al, 2017)。结构稳定性 试图从群落的各个层面(包括群落结构、群落子结构 (sub-community)和单种群等)来厘清这个关系。

第一, 研究群落结构和物种共存的关系。多个 研究阐明了不同的种间关系(随机、互利和竞争)的 群落结构对物种共存的影响。对于随机的群落结构, 研究给出了结构稳定性的解析解(Grilli et al, 2017)。 这一随机群落结构的解析解可以用来衡量非随机 群落结构对结构稳定性的贡献。对于互利的非随机 群落结构, 研究发现嵌套性可以增加群落的结构稳 定性(Rohr et al, 2014; Pascual-García \& Bastolla, 2017)。对于竞争的非随机群落结构, 研究发现, 群 落的结构稳定性会随着种内竞争的增强而升高, 而 且结构稳定性的最大值和群落结构无关(Barabás et al, 2016)。这一理论工作将现代共存理论的核心结 论推广到了多物种群落(Chesson, 2000; Barabás et al, 2018; Song et al, 2019a)。另外, 对于竞争的非随 


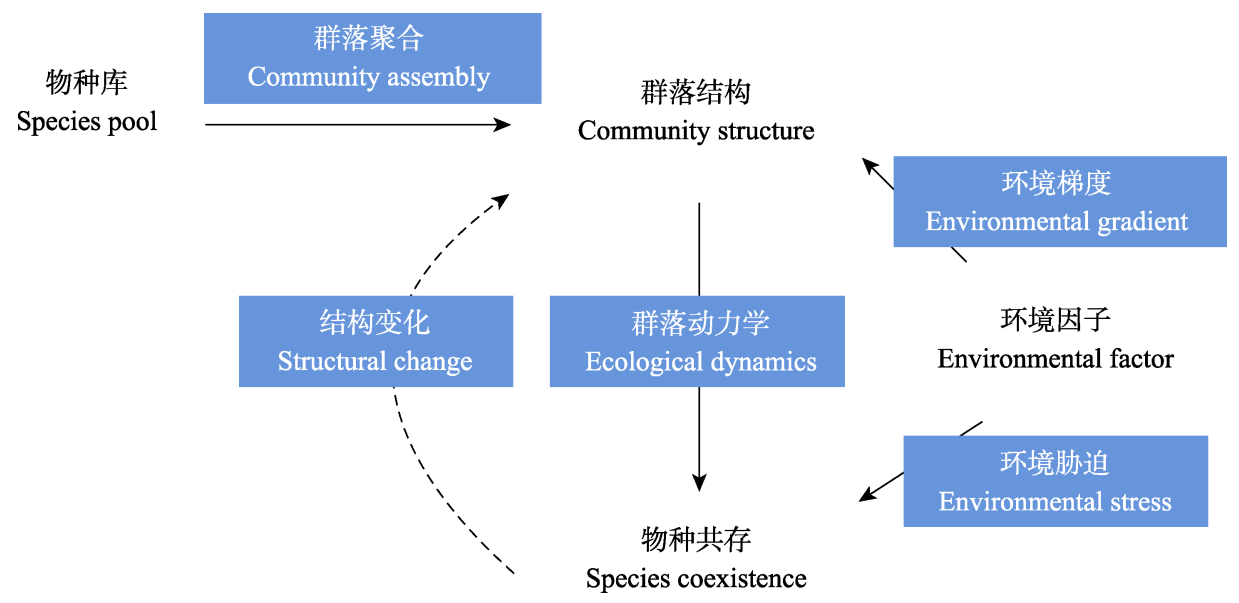

图4 结构稳定性理论的物种共存框架。群落中有4个核心因素: 物种库、群落结构、环境因子和物种共存。这些核心因素之 间都有着具体的生态过程(蓝色): 群落聚合、结构变化、群落动力学、环境梯度和环境胁迫。结构稳定性理论试图将这些生 态过程整合在一个统一的框架下。对于图中的每一个生态过程, 结构稳定性理论都提出了新的假说和验证方法, 并在实验以 及观测数据中得到了验证。

Fig. 4 The structural stability approach for understanding multispecies coexistence. The nodes denote the key elements in ecological dynamics: species pool, community structure, environmental factors, and species coexistence. The links represent the ecological processes (denoted in blue) that connect these elements: community assembly, structural change, ecological dynamics, environmental gradient, and environmental stress. The structural stability approach aims to integrate these ecological processes under a unified framework. For each process, the structural stability approach provides new theoretical predictions, which are validated by experimental and/or observational data.

机群落结构, 研究发现群落的结构稳定性在大多数 情况下随着种内差异(intraspecific variation)的增加 而减少(Barabás \& D’Andrea, 2016)。这一理论工作 和其他关于种内差异的理论工作的定性结果是相 同的(Hart et al, 2016; Des Roches et al, 2018)。

第二, 多物种群落和少物种群落的共存条件的 区别。由于多物种导致的间接作用 (indirect interaction), 少物种群落和多物种群落的共存条件 (即结构稳定性的共存域) 不一定相同 (Wootton, 1994)。少物种群落和多物种群落的物种共存条件可 分为3类(Saavedra et al, 2017b): (1)少物种群落的共 存条件全都是多物种群落的共存条件(即少物种群 落的共存域是多物种群落的共存域的子集); (2)少 物种群落的共存条件只有一部分是多物种群落的 共存条件(即少物种群落的共存域和多物种群落的 共存域有一部分重叠); (3)少物种群落的共存条件 全都不是多物种群落的共存条件(即少物种群落的 共存域和多物种群落的共存域没有重叠)。这一分类 在很多经典的物种共存理论中是很难做到的 (Levine et al, 2017)。基于一年生植物的竞争实验研 究发现, $80 \%$ 的一年生植物群落都是第二类, 也就 是少物种群落的共存域和多物种群落的共存域只
有一部分交叉(Saavedra et al, 2017b)。后续更深入的 研究发现, 这一现象的产生可能是因为动物作用 (如蚂蚁)对一年生植物的影响(Petry et al, 2018)。

第三, 如何衡量特定物种对物种共存的重要 性。一个群落内不同的物种对群落共存的贡献是不 同的。粗略而言, 对于贡献小的物种, 群落在失去 这一物种后剩余的物种可以继续共存; 而对于贡献 大的物种, 群落在失去这一物种后剩余的物种则难 以共存。特别地, 那些对群落延续至关重要的物种 被称为关键种(keystone species)。然而, 如何去定量 刻画某个特定物种的贡献并不容易(Power et al, 1996)。在结构稳定性理论中, 特定物种对共存的贡 献定义为群落中存在这一物种的结构稳定性和群 落中没有这一物种的结构稳定性之间的差别(Cagua et al, 2019; Simmons et al, 2019)。这一简单的定义可 以帮助我们定量地研究关键种和群落的其他性质 的关系。基于这一定义, 近期研究发现互利群落的 关键种同时也是复杂网络中的(根据结构可控性理 论)驱动节点(Cagua et al, 2019), 因而关键种对群落 的可控性有着至关重要的影响(Liu et al, 2011)。同 样基于这一定义, 另一研究则发现互利群落的关键 种同时也是最容易灭绝的(Simmons et al, 2019), 因 
而关键种可能是十分脆弱的(Saavedra et al, 2011)。 重要的是, 这一定义的应用不仅仅局限于对关键种 的量化。比如, 我们可以通过类似的办法来量化单 一入侵物种对物种共存的影响(Godoy, 2019)。

\section{2 环境变化如何影响群落结构}

环境梯度(environmental gradient)在环境因子 和群落结构之间扮演了联结性的角色。结构稳定性 理论试图理解群落结构和种间关系会如何随着环 境梯度而变化。

第一, 嵌套性结构的强弱如何随着环境梯度而 变化。理论工作证明了互利群落结构的嵌套性越强, 其结构稳定性越高(Rohr et al, 2014)。但是，这并不 意味着真实的互利群落都应该有很强的嵌套性。互 利群落分布在各种地区, 而不同地区的环境变化程 度各有高低。所以, 只有在环境波动强烈地区的互 利群落才需要更高的结构稳定性。反过来, 可以预 测, 处于环境剧烈变化的地区的互利群落具有嵌套 性更强的群落结构。实证数据很好地支持了这一假 设(Song et al, 2017, 2019b)。后续的研究发现这一定 性结果在其他实证数据中也成立(Fontenla et al, 2019; Classen et al, 2020)。这一结果解释了为什么观 测到的互利群落的嵌套性通常并不太强的现象 (Staniczenko et al, 2013; Payrató-Borràs et al, 2019)。

第二, 互利群落和食物网的结构区别。如前文 所提到的, 不少生态学家认为嵌套性和模块化是区 别互利群落和食物网的结构特征(Bascompte, 2010; Thébault \& Fontaine, 2010; Delmas et al, 2019)。然而, 利用全面的实证数据和严格的统计方法, 最近有研 究发现互利群落和食物网的嵌套性或者模块化的 程度并没有显著差异(Michalska-Smith \& Allesina, 2019), 而且在其他常见的网络结构的度量上也没 有显著差异。这一研究的直接推论是群落结构和物 种共存之间没有直接关联, 挑战了生态网络的研究 基石。然而, 这一研究并没有控制不同区域的环境 变化程度。新的研究发现互利群落和食物网对群落 稳定性的度量(包括结构稳定性)在环境梯度上有着 显著的相反趋势(Song \& Saavedra, 2020b)。和上文 提及的嵌套性结构的强弱一样, 这一结果同样突显 了环境因子在群落结构的研究中的重要性。

第三，种间关系强弱如何随环境因素而变化。 环境因素会对物种之间的相互关系造成影响, 甚至
转变种间关系的类别(例如从互利到竞争)。这一问 题被称为种间关系的情境依赖(context-dependency of species interactions) (Chamberlain et al, 2014)。对 于这一问题, 最经典的生态学理论是胁迫梯度假说 (stress gradient hypothesis): 在低胁迫环境中植物间 主要是竞争关系, 而在高胁迫环境中则主要是促进 关系(Callaway et al, 2002; 张炜平等, 2013; Hoek et al, 2016)。然而, 这些经典理论大多都是定性的而非 定量的。结构稳定性理论则可以为此提供定量研究 框架(Song et al, 2020b)。这一新的理论框架可以通 过群落结构和环境条件来计算种间关系转变的概 率。例如, 在固定的环境条件下(例如实验室实验), 转变概率等于转变前和转变后的群落的共存域的 交集相对于转变前的共存域的相对大小, 而在变化 较大的环境条件下(例如野外观测)转变概率等于转 变后的群落的结构稳定性。这一理论解释了一个互 利研究中的重要悖论: 为什么互利关系在自然界中 广泛存在(Frederickson, 2017), 但在实验中却最容 易转变为其他类型的种间关系(Chamberlain et al, 2014)。

\section{3 环境胁迫如何影响物种共存}

环境胁迫在环境因子和物种共存关系中扮演 了联结性的角色。环境胁迫在这里特指环境变化对 物种共存的影响。结构稳定性理论试图阐释环境胁 迫如何影响物种共存。

第一, 环境胁迫影响了物种共存的哪些相关过 程。具体而言, 环境胁迫更容易通过影响群落中物 种的内禀增长率还是通过影响群落结构来影响物 种共存? 如前所述, 我们可以定义内禀增长率的结 构稳定性(Song et al, 2018b)和群落结构的结构稳定 性(Chesson, 2018)。重要的是，内禀增长率的结构稳 定性和群落结构的结构稳定性具有权衡关系 (trade-off) (Song et al, 2020a)。也就是说，增加内禀 增长率的结构稳定性会减少群落结构的结构稳定 性，反之亦然。通过群落的实际观察得到的参数， 我们就可以发现群落更趋向于提高内禀增长率的 结构稳定性还是群落结构的结构稳定性, 然后就可 以推测出这个群落所受到的环境胁迫是在内禀增 长率上还是在群落结构上。利用加利福利亚州的 18种一年生植物的竞争实验数据(Godoy et al, 2014), Song等(2020a)发现一年生植物群落倾向于最大化 内禀增长率的结构稳定性而不是群落结构的结构 
稳定性。这意味着一年生植物群落受到的环境胁迫 更可能作用在内禀增长率上。

第二, 哪些环境胁迫因子影响了物种共存。如 前文提及的, 我们可以从种群多度的时间序列中直 接估计群落在任一时刻的局部结构稳定性(Cenci \& Saavedra, 2019)。局部结构稳定性是较为抽象的统 计学概念, 并没有全局结构稳定性的可解释性(即 物种共存的概率)。但是, 局部结构稳定性可以更好 地利用统计学方法来研究环境因子对群落的影响 (Cenci \& Saavedra, 2019; Cenci et al, 2020; Song \& Saavedra, 2020a)。比如, 通过比较局部结构稳定性 的时间序列和环境因子的时间序列(比如温度和湿 度等等), 我们就可以推测出到底是哪些环境因子 驱动着群落的变化(Sugihara et al, 2012; Deyle et al, 2016a; Nova et al, 2019)。基于该方法, 研究发现温 度(而非海浪高度)是岩礁潮间带群落的局部结构稳 定性的主导因子(Benincà et al，2015; Cenci \& Saavedra, 2019)。更具体而言, 温度引起了群落结构 的改变, 而结构变化引起了结构稳定性的变化。

\section{4 群落结构变化的规律}

群落结构并不是固定不变的, 而是会随物种能 否共存而发生变化。结构稳定性理论试图理解和预 测群落结构的变化。

第一, 理解结构变化和强季节性的环境变化的 关系。生态学假说认为处于强季节性的环境中的群 落会尽可能维持稳态(homeostasis) (Odum, 1969; Morgan Ernest \& Brown, 2001)。然而, 在不同季节 中群落的物种组成会有很大变化, 群落维持稳态的 机制是什么呢? 一个可能的假设是群落结构在不 同季节会发生重组(reorganization)。利用在比亚沃韦 扎原始森林的夏季和秋季的猎物一捕食者群落, Saavedra等(2016a)发现群落结构的重组并不是随机 的, 而是尽可能使在不同季节里群落的结构稳定性 变化较小。这一结果支持了群落重组是维持群落稳 态的一个机制。

第二, 预测结构变化和物候现象的关系。一个 群落的物种组成并不是固定的, 其中物种出现和消 失的时间是物候现象的组成部分。物候现象一般被 认为是由环境因素(例如温度和降水)决定的(陆佩 玲等, 2006; Forrest \& Thomson, 2011)。然而, 物候 现象也会受到群落结构的影响: 群落的结构稳定性
越小，物种就更有可能消失，反之亦然。利用在格 陵兰岛上的一个植物-传粉者群落的时序数据(每天 的物候现象、群落结构和环境因素), Song和 Saavedra (2018a)发现结构稳定性可以比环境因素 更好地预测物候现象。

\section{5 群落聚合的规律}

群落聚合(community assembly)在物种库和群 落结构关系中扮演了联结性的角色。每个群落都有 着其独特的聚合历史。结构稳定性理论试图破译群 落的聚合规则, 以及理解群落聚合和群落演替的 关系。

第一，破解群落的聚合规则(assembly rule)。优 先效应(priority effects)指的是物种出现的先后顺序 会影响群落的结构乃至物种共存(Fukami, 2015)。很 多研究试图理解在短时间尺度中物种出现的顺序 是如何影响群落的(Chase，2003; Fukami，2015; Sprockett et al, 2018)。在短时间尺度上，研究发现生 态位优先占领 (niche preemption)和生态位改造 (niche modification)是两种重要的产生优先效应的 生态学机理(Fukami, 2015)。然而, 哪个生态学机理 在长时间尺度上更加重要呢? 鉴于在长时间尺度 上开展实验非常困难，结构稳定性可以作为一个辅 助工具来研究优先效应。利用中欧一个地区长达 2000年的入侵植物的先后顺序, Song等(2018a)发现 这一植物-草食动物群落的结构稳定性伴随着演替 有明显的增强。更进一步地, 研究发现生态位优先 占领，而不是生态位改造，导致了这一植物-草食 动物群落的结构稳定性的增强。换言之, 这一植物一 草食动物的聚合规则是同一科(而不是不同科)的植 物先来后到的顺序。

第二, 破解群落演替和群落聚合的关系。一般 而言，群落随着演替愈发成熟，物种库的新物种会 更难定居到这一群落(Margalef, 1968; Odum, 1969)。 这一生态学现象是如何产生的呢? 利用热带季节 干旱林多年的次生演替数据, Saavedra等(2017a)研 究发现演替的一个重要标志是群落结构的变化。具 体而言, 通过群落结构的变化, 居留种 (resident species)在群落的演替中不断提高着群落的结构稳 定性, 并且在演替的后期阻碍迁徙种 (colonizing species)的定殖。 


\section{4 研究展望}

利用结构稳定性这一工具, 我们可以系统地整 合物种库、群落结构、环境因子等因素来研究物种 共存。当然, 结构稳定性理论在群落生态学中只有 短短几年的发展, 因而在理论层面和实证层面都有 着很多问题值得进一步探索。下面从 4 个方面探讨 未来的一些发展可能性。

\section{1 群落结构的特异性研究}

最早的群落结构思想可以追溯到达尔文所提 出 的 “entangled bank” 的这一绝妙概念 (Darwin, 1859)。在Robert May于20世纪70年代奠基性的工作 之后(May，1972，1975), 现代群落生态学就开始系 统地研究群落结构和物种共存的关系(May, 2006)。

例如, May (1972)中提出的模块化结构就成为食物 网研究中经久不衰的话题(Pimm, 1982; Solow et al, 1999; Stouffer \& Bascompte, 2011; Grilli et al, 2016; Cenci et al, 2018b; Nordbotten et al, 2018)。伴随着更 多群落结构的数据和复杂性科学的兴起(Pascual \& Dunne, 2006; Newman, 2010), 理论生态学家更是系 统地研究了普适结构。遗憾的是, 相较于对普适结 构的关注, 理论生态学家对群落结构的特异性的关 注少了很多。然而, 实证数据清楚地表明群落结构 在时间尺度(CaraDonna et al, 2017)和空间尺度 (Song et al, 2017)上都有着不可忽略的变化。

当然, 这一现象并不仅仅存在于群落生态学的 研究中, 类似的现象同样存在于复杂性科学中。例 如, 最近不少复杂网络科学家争论无尺度网络 (scale-free network) 究竟是不是现实网络的一个普 适结构(Voitalov et al, 2018; Broido \& Clauset, 2019; Gerlach \& Altmann, 2019; Holme, 2019)。这一现象 的深层原因可能是理论生态学的研究者往往有着 深厚的数学或者物理学背景(Egler, 1986)。但正如复 杂网络科学开始重视时序网络(temporal network) (Li et al, 2017), 新的群落结构的研究范式中需要研 究群落结构的普适性和特异性之间的张力(Alberch, 1989)。

结构稳定性理论的核心出发点是将环境因子 融入到群落结构特异性的研究中。然而, 环境因子 只是群落结构特异性的决定因素之一。例如, 生活 史(Woodward et al, 2005; Ings et al, 2009)和人类活 动(Yeakel et al, 2014)都是不可忽视的决定因素。未
来可能的一个重要方向是应用结构稳定性或者建 立新的理论工具来将这些决定因素整合到群落结 构的研究中。

\section{2 物种共存的概念拓展}

类似于其他的许多物种共存的概念，结构稳定 性这一概念关注的是全部物种都可以共存。然而, 实际研究中很多时候关心的问题是多少物种能共 存, 而不拘泥于一个都不能少。那么, 结构稳定性 理论是否还适用呢? 现有的结构稳定性理论可以 作为一个近似的衡量群落中结构变化的度量: 当群 落的结构稳定性越小以及环境变化程度越大, 物种 就更有可能离开或者群落结构更容易发生变化, 反 之亦然。如前文所述, 这一理论预测有着许多实证 数据支撑(Saavedra et al, 2016a; Song \& Saavedra, 2018a)。但这一理论预测较为粗楉, 我们需要进一 步地拓展现有的结构稳定性概念。一个可能的拓展 是定量计算 $m$ 个物种的物种库中 $n$ 个物种共存的结 构稳定性(现有的结构稳定性考虑的是 $m=n$ 的情 况)。另一个可能的拓展是定量计算 $m$ 个物种的物种 库中每一个物种存活的概率(现有的结构稳定性考 虑的是每一个物种共存的概率相同, 类似于平均场 近似)。具体而言, 考虑物种库中存在 3 个物种甲、 乙和丙，那么甲物种存活的概率等于甲单独存活、 甲和乙共存、甲和丙共存、以及甲、乙和丙都共存 这4种情况的概率之和。

\section{3 建立和其他理论的联系}

结构稳定性理论只是完整的物种共存的宏大 理论的一小块拼图。不同的生态学理论彼此和而不 同(牛克昌等, 2009), 因此我们需要更好地将结构稳 定性和其他理论工作结合在一起。

第一, 建立结构稳定性和其他共存理论的联 系。过去的一些研究将结构稳定性和一些经典的共 存理论建立了联系, 包括生态位理论(Godoy et al, 2018)、随机矩阵理论(Grilli et al, 2017)、消费者一资 源理论(Butler \& O’Dwyer, 2018)和现代共存理论 (Song et al, 2020a)。然而, 近几年理论群落生态学的 迅猛发展, 产生了很多新的理论。例如, 随机群落 的聚合理论(Bunin, 2017; Serván et al, 2018; Wang, 2018)、敏感度分析理论(Barabás et al, 2014a, b)和性 状聚类理论(D’Andrea et al, 2018, 2019)等等。将这 些新的理论工具和结构稳定性联系起来可以帮助 我们更全面地理解物种共存研究的当前图景。 
第二, 建立结构稳定性和其他群落生态学问题 的联系。尽管物种共存是群落生态学的核心问题之 一, 群落生态学还有着很多其他的核心问题。比如, 另一个核心问题是生物多样性和生态系统功能之 间的关系(Loreau et al, 2001; Wang \& Brose, 2018)。 结构稳定性理论初步地将群落结构更系统地整合 进生物多样性和生态系统功能的研究中(Rohr et al, 2016)。但是，结构稳定性理论并没有和生物多样性 和生态系统功能的相关研究很好地嵌合(例如互补 效应及抽样效应, Loreau \& Hector, 2001)。再比如, 还有一个核心问题是群落和集合群落(metacommunity)的关系(Leibold \& Chase, 2017; Guzman et al, 2019; Wang et al, 2019)。目前只有初步的理论工作 将结构稳定性推广到集合群落(Arumugam et al, 2019)。更为系统地将群落层面上的结构稳定性理论 推广到集合群落层面上可以更好地整合群落生态 学中不同的基本过程(朱璧如和张大勇, 2011; Vellend, 2016)。

第三，建立结构稳定性和其他领域的理论的联 系。理论生态学需要吸收别的领域的知识。特别是 复杂网络科学, 是一个有很大借鉴价值的领域。在 复杂网络科学刚刚兴起的时候, 理论生态学家就借 鉴了非标度网络的概念来研究群落结构(Dunne et al, 2002)。一个值得借鉴的理论是复杂网络科学的 结构可控性理论(structural controllability), 研究的 是网络结构如何决定复杂网络的可控性(Liu et al, 2011; Liu \& Barabási, 2016)。尽管已有研究将结构 可控性理论和结构稳定性建立了初步联系(Arnoldi \& Haegeman, 2016; Cagua et al, 2019), 但还是落后 于可控性理论的最新进展( $\mathrm{Li}$ et al, 2017; Angulo et al, 2019)。另一个值得借鉴的理论是复杂网络科学 的多层网络(multi-layer network), 研究的是处于不 同层级但相互关联的网络。越来越多的实证证据指 出生态学群落的结构也是多层网络(Pilosof et al, 2017; Hutchinson et al, 2019)。然而, 目前还没有工 作将结构稳定性运用于多层的群落结构。

\section{4 更稳健的实证支撑}

生态学本质上是实证科学, 没有实证数据支撑 的理论就像一盘散沙。目前, 尽管结构稳定性理论 有着一定的经验证据的支撑, 但是这些经验证据来 自于不同类型的生态群落。

比如, 植物-传粉者群落(Song et al, 2017)、植
物-蚂蚁群落(Petry et al, 2018)、植物 - 草食动物群落 (Song et al, 2018a)、岩礁相潮间带群落(Cenci \& Saavedra, 2019)、一年生植物群落(Song et al, 2020a) 和真菌-宿主群落(Gomes et al, 2017)等。即使是在 同类型的生态群落中, 实证数据的类型也有不少细 微的差别(Saavedra et al, 2016b; Song et al, 2017; Cenci et al, 2018a)。因此, 结构稳定性理论还需要更 为系统和全面的实证证据的支撑(或者证伪)。一方 面，结构稳定性理论提供了许多新的理论假说，因 而可以指导新的实验。比如，结构稳定性理论可以 帮助设计诸如如何比较种间关系的情境依赖性 (Song et al, 2020b)、如何量化群落中的关键种 (Cagua et al, 2019), 以及如何在实验中验证高阶物 种作用的存在(AlAdwani \& Saavedra, 2019)等生态 学问题。

另一方面，结构稳定性理论提供了一套完整的 检验方法，因而也可以直接运用于许多已经采集到 的实证数据。例如, 加利福尼亚州一年生植物的竞 争实验最初是基于现代研究理论分析的(Godoy et al, 2014; Kraft et al, 2015)。将结构稳定性理论运用 在这个一年生植物群落的实验数据中, 研究揭示了 其新的生态学性质(Saavedra et al, 2017b; Song et al, 2020a)。类似地，我们不难把结构稳定性理论运用 于别的实证数据(比如检验现代共存理论的许多实 验数据)。研究表明, 从单一的群落实验中很难确定 物种共存的机理, 而是需要综合不同的群落实验 (Clark et al, 2019)。因而，这一个方向的工作可以更 稳健地验证物种共存的普适原理。

\section{结语}

结构稳定性理论是一个试图在群落层面上理 解和预测物种共存的理论框架。这一理论可以定量 地根据群落结构和环境因子计算出物种共存的概 率。在此基础上, 这一理论系统地建立了物种库、 群落结构、环境因子和物种共存之间的关系，并对 这些关系一一提出了新的理论预测以及提供了对 应的实证证据。结构稳定性理论弥合了当前理论和 实践之间的部分鸿沟, 有利于更好地理解物种共存 的基本原理。广泛而言，这一理论相对灵活，可以 因地制宜地对所研究的群落建模。因此, 结构稳定 性理论有望成为比较和综合不同生态学群落的“通 用货币”。现有的结构稳定性理论远未成熟，在理论 
层面以及实证层面都还有着许多的研究空间和机 会, 希望本文有助于国内生态学工作者了解和应用 这一理论。

\section{参考文献}

Adler PB, HilleRisLambers J, Levine JM (2007) A niche for neutrality. Ecology Letters, 10, 95-104.

AlAdwani M, Saavedra S (2019) Is the addition of higher-order interactions in ecological models increasing the understanding of ecological dynamics? Mathematical Biosciences, 315, 108222.

Alberch P (1989) The logic of monsters: Evidence for internal constraint in development and evolution. Geobios, 22, 21-57.

Allesina S, Tang S (2012) Stability criteria for complex ecosystems. Nature, 483, 205-208.

Angulo MT, Moog CH, Liu YY (2019) A theoretical framework for controlling complex microbial communities. Nature Communications, 10, 1-12.

Arnoldi JF, Haegeman B (2016) Unifying dynamical and structural stability of equilibria. Proceedings of the Royal Society A: Mathematical, Physical and Engineering Sciences, 472, 20150874.

Azaele S, Suweis S, Grilli J, Volkov I, Banavar JR, Maritan A (2016) Statistical mechanics of ecological systems: Neutral theory and beyond. Reviews of Modern Physics, 88, 35003.

Barabás G, D’Andrea R (2016) The effect of intraspecific variation and heritability on community pattern and robustness. Ecology Letters, 19, 977-986.

Barabás G, D’Andrea R, Stump SM (2018) Chesson's coexistence theory. Ecological Monographs, 88, 277-303.

Barabás G, Michalska-Smith MJ, Allesina S (2016) The effect of intra- and interspecific competition on coexistence in multispecies communities. The American Naturalist, 188, E1-E12.

Barabás G, Meszéna G, Ostling A (2014a) Fixed point sensitivity analysis of interacting structured populations. Theoretical Population Biology, 92, 97-106.

Barabás G, Pásztor L, Meszéna G, Ostling A (2014b) Sensitivity analysis of coexistence in ecological communities: Theory and application. Ecology Letters, 17, 1479-1494.

Bascompte J (2010) Structure and dynamics of ecological networks. Science, 329, 765-766.

Bascompte J, Jordano P, Melián CJ, Olesen JM (2003) The nested assembly of plant-animal mutualistic networks. Proceedings of the National Academy of Sciences, USA, 100, 9383-9387.

Bascompte J, Jordano P, Olesen JM (2006) Asymmetric coevolutionary networks facilitate biodiversity maintenance. Science, 312, 431-433.

Bastolla U, Fortuna MA, Pascual-García A, Ferrera A, Luque B, Bascompte J (2009) The architecture of mutualistic networks minimizes competition and increases biodiversity. Nature, 458, 1018-1020.

Bastolla U, Lässig M, Manrubia SC, Valleriani A (2005) Biodiversity in model ecosystems. I. Coexistence conditions for competing species. Journal of Theoretical Biology, 235, 521-530.

Bažant ZP (2000) Structural stability. International Journal of Solids and Structures, 37, 55-67.

Benincà E, Ballantine B, Ellner SP, Huisman J (2015) Species fluctuations sustained by a cyclic succession at the edge of chaos. Proceedings of the National Academy of Sciences, USA, 112, 6389-6394.

Broido AD, Clauset A (2019) Scale-free networks are rare. Nature Communications, 10, 1017.

Bunin G (2017) Ecological communities with Lotka-Volterra dynamics. Physical Review E, 95, 42414.

Butler S, O’Dwyer JP (2018) Stability criteria for complex microbial communities. Nature Communications, 9, 2970.

Cagua EF, Wootton KL, Stouffer DB (2019) Keystoneness, centrality, and the structural controllability of ecological networks. Journal of Ecology, 107, 1779-1790.

Callaway RM, Brooker R, Choler P, Kikvidze Z, Lortie CJ, Michalet R, Paolini L, Pugnaire FI, Newingham B, Aschehoug ET, Armas C, Kikodze D, Bradley JC (2002) Positive interactions among alpine plants increase with stress. Nature, 417, 844-848.

CaraDonna PJ, Petry WK, Brennan RM, Cunningham JL, Bronstein JL, Waser NM, Sanders NJ (2017) Interaction rewiring and the rapid turnover of plant-pollinator networks. Ecology Letters, 20, 385-394.

Case TJ (2000) Illustrated Guide to Theoretical Ecology. Oxford University Press, Oxford.

Cenci S, Medeiros LP, Sugihara G, Saavedra S (2020) Assessing the predictability of nonlinear dynamics under smooth parameter changes. Journal of the Royal Society Interface, 17, 20190627.

Cenci S, Montero-Castaño A, Saavedra S (2018a) Estimating the effect of the reorganization of interactions on the adaptability of species to changing environments. Journal of Theoretical Biology, 437, 115-125.

Cenci S, Saavedra S (2018) Structural stability of nonlinear population dynamics. Physical Review E, 97, 12401.

Cenci S, Saavedra S (2019) Non-parametric estimation of the structural stability of non-equilibrium community dynamics. Nature Ecology and Evolution, 3, 912-918.

Cenci S, Song C, Saavedra S (2018b) Rethinking the importance of the structure of ecological networks under an environment-dependent framework. Ecology and Evolution, 8, 6852-6859.

Cenci S, Sugihara G, Saavedra S (2019) Regularized S-map for inference and forecasting with noisy ecological time series. Methods in Ecology and Evolution, 10, 650-660.

Chamberlain SA, Bronstein JL, Rudgers JA (2014) How con- 
text dependent are species interactions? Ecology Letters, 17, 881-890.

Chase JM (2003) Community assembly: When should history matter? Oecologia, 136, 489-498.

Chase JM, Leibold MA (2003) Ecological Niches: Linking Classical and Contemporary Approaches. University of Chicago Press, Chicago.

Chesson P (2000) Mechanisms of maintenance of species diversity. Annual Review of Ecology, Evolution, and Systematics, 31, 343-366.

Chesson P (2018) Updates on mechanisms of maintenance of species diversity. Journal of Ecology, 106, 1773-1794.

Chu CJ, Wang YS, Liu Y, Jiang L, He FL (2017) Advances in species coexistence theory. Biodiversity Science, 25, 345-354. (in Chinese with English abstract) [储诚进, 王西 石, 刘宇, 蒋林, 何芳良 (2017) 物种共存理论研究进展. 生物多样性, 25, 345-354.]

Clark A, Hillebrand H, Harpole WS (2019) Scale both confounds and informs characterization of species coexistence in empirical systems. The American Naturalist, 194, 794-806.

Classen A, Eardley CD, Hemp A, Peters MK, Peters RS, Ssymank A, Steffan-Dewenter I (2020) Specialization of plant-pollinator interactions increases with temperature at Mt. Kilimanjaro. Ecology and Evolution, 10, 2182-2195.

Coulson T, Kendall BE, Barthold J, Plard F, Schindler S, Ozgul A, Gaillard JM (2017) Modeling adaptive and nonadaptive responses of populations to environmental change. The American Naturalist, 190, 313-336.

Currie DJ (2019) Where Newton might have taken ecology. Global Ecology and Biogeography, 28, 18-27.

D’Andrea R, Ostling A, O’Dwyer JP (2018) Translucent windows: How uncertainty in competitive interactions impacts detection of community pattern. Ecology Letters, 21, 826-835.

D’Andrea R, Riolo M, Ostling AM (2019) Generalizing clusters of similar species as a signature of coexistence under competition. PLoS Computational Biology, 15, e1006688.

Darwin C (1859) On the Origin of Species, p. 62. John Murray, London.

Delmas E, Besson M, Brice MH, Burkle LA, Dalla Eiva GV, Fortin MJ, Gravel D, Guimarães PR Jr, Hembry DH, Newman EA, Olesen JM, Pires MM, Yeakel JD, Poisot T (2019) Analysing ecological networks of species interactions. Biological Reviews, 94, 16-36.

Des Roches S, Post DM, Turley NE, Bailey JK, Hendry AP, Kinnison MT, Schweitzer JA, Palkovacs EP (2018) The ecological importance of intraspecific variation. Nature Ecology and Evolution, 2, 57-64.

Deyle ER, Maher MC, Hernandez RD, Basu S, Sugihara G (2016a) Global environmental drivers of influenza. Proceedings of the National Academy of Sciences, USA, 113,
13081-13086.

Deyle ER, May RM, Munch SB, Sugihara G (2016b) Tracking and forecasting ecosystem interactions in real time. Proceedings of the Royal Society B: Biological Sciences, 283, 20152258.

Dougoud M, Vinckenbosch L, Rohr RP, Bersier LF, Mazza C (2018) The feasibility of equilibria in large ecosystems: A primary but neglected concept in the complexity-stability debate. PLoS Computational Biology, 14, e1005988.

Dunne JA, Williams RJ, Martinez ND (2002) Food-web structure and network theory: The role of connectance and size. Proceedings of the National Academy of Sciences, USA, 99, 12917-12922.

Egler FE (1986) "Physics Envy" in ecology. Bulletin of the Ecological Society of America, 67, 233-235.

Fontenla JL, Fontenla Y, Cuervo Z, Álvarez de Zayas A (2019) Red de interacción ecológica insectos-plantas en playas del este, la habana, cuba. Acta Botánica Cubana, 218, 129-142.

Forrest JR, Thomson JD (2011) An examination of synchrony between insect emergence and flowering in Rocky Mountain meadows. Ecological Monographs, 81, 469-491.

Fort H, Vázquez DP, Lan BL (2016) Abundance and generalisation in mutualistic networks: Solving the chicken-and-egg dilemma. Ecology Letters, 19, 4-11.

Frederickson ME (2017) Mutualisms are not on the verge of breakdown. Trends in Ecology and Evolution, 32, 727-734.

Fukami T (2015) Historical contingency in community assembly: Integrating niches, species pools, and priority effects. Annual Review of Ecology, Evolution, and Systematics, 46, $1-23$.

Garlaschelli D, Caldarelli G, Pietronero L (2003) Universal scaling relations in food webs. Nature, 423, 165-168.

Genz A, Bretz F (2002) Comparison of methods for the computation of multivariate $t$ probabilities. Journal of Computational and Graphical Statistics, 11, 950-971.

Gerlach M, Altmann EG (2019) Testing statistical laws in complex systems. Physical Review Letters, 122, 168301.

Godoy O (2019) Coexistence theory as a tool to understand biological invasions in species interaction networks: Implications for the study of novel ecosystems. Functional Ecology, 33, 1190-1201.

Godoy O, Bartomeus I, Rohr RP, Saavedra S (2018) Towards the integration of niche and network theories. Trends in Ecology and Evolution, 33, 287-300.

Godoy O, Kraft NJ, Levine JM (2014) Phylogenetic relatedness and the determinants of competitive outcomes. Ecology Letters, 17, 836-844.

Gomes SI, Merckx VS, Saavedra S (2017) Fungal-host diversity among mycoheterotrophic plants increases proportionally to their fungal-host overlap. Ecology and Evolution, 7, 3623-3630.

Grilli J, Adorisio M, Suweis S, Barabás G, Banavar JR, Allesina S, Maritan A (2017) Feasibility and coexistence of 
large ecological communities. Nature Communications, 8, 14389.

Grilli J, Rogers T, Allesina S (2016) Modularity and stability in ecological communities. Nature Communications, 7, 1-10.

Guimarães PR, Pires MM, Jordano P, Bascompte J, Thompson JN (2017) Indirect effects drive coevolution in mutualistic networks. Nature, 550, 511-514.

Guzman LM, Germain RM, Forbes C, Straus S, O’Connor MI, Gravel D, Srivastava DS, Thompson PL (2019) Towards a multi-trophic extension of metacommunity ecology. Ecology Letters, 22, 19-33.

Hart SP, Schreiber SJ, Levine JM (2016) How variation between individuals affects species coexistence. Ecology Letters, 19, 825-838.

Harte J (2011) Maximum Entropy and Ecology: A Theory of Abundance, Distribution, and Energetics. Oxford University Press, Oxford.

Harte J, Newman EA (2014) Maximum information entropy: A foundation for ecological theory. Trends in Ecology and Evolution, 29, 384-389.

Hoek TA, Axelrod K, Biancalani T, Yurtsev EA, Liu J, Gore J (2016) Resource availability modulates the cooperative and competitive nature of a microbial cross-feeding mutualism. PLoS Biology, 14, e1002540.

Hofbauer J, Sigmund K (1998) Evolutionary Games and Population Dynamics. Princeton University Press, Princeton.

Holland JN, Okuyama T, DeAngelis DL (2006) Comment on "asymmetric coevolutionary networks facilitate biodiversity maintenance”. Science, 313, 1887.

Holme P (2019) Rare and everywhere: Perspectives on scale-free networks. Nature Communications, 10, 1016.

Hubbell SP (1997) A unified theory of biogeography and relative species abundance and its application to tropical rain forests and coral reefs. Coral Reefs, 16, S9-S21.

Hutchinson GE (1978) An Introduction to Population Ecology. Yale University Press, New Haven.

Hutchinson MC, Bramon Mora B, Pilosof S, Barner AK, Kéfi S, Thébault E, Jordano P, Stouffer DB (2019) Seeing the forest for the trees: Putting multilayer networks to work for community ecology. Functional Ecology, 33, 206-217.

Ings TC, Montoya JM, Bascompte J, Blüthgen N, Brown L, Dormann CF, Edwards F, Figueroa D, Jacob U, Jones JI, Lauridsen RB, Ledger ME, Lewis HM, Olesen JM, Van Veen FJ, Warren PH, Woodward G (2009) Ecological networks-Beyond food webs. Journal of Animal Ecology, 78, 253-269.

James A, Pitchford JW, Plank MJ (2012) Disentangling nestedness from models of ecological complexity. Nature, 487, 227-230.

James A, Pitchford JW, Plank MJ (2013) James et al reply. Nature, 500, E2-E3.

Jansen V, Sigmund K (1998) Shaken not stirred: On permanence in ecological communities. Theoretical Population
Biology, 54, 195-201.

Kraft NJ, Godoy O, Levine JM (2015) Plant functional traits and the multidimensional nature of species coexistence. Proceedings of the National Academy of Sciences, USA, 112, 797-802.

Lawton JH (1999) Are there general laws in ecology? Oikos, 84, 177-192.

Leibold MA, Chase JM (2017) Metacommunity Ecology. Princeton University Press, Princeton.

Letten AD, Ke PJ, Fukami T (2017) Linking modern coexistence theory and contemporary niche theory. Ecological Monographs, 87, 161-177.

Letten AD, Stouffer DB (2019) The mechanistic basis for higher-order interactions and nonadditivity in competitive communities. Ecology Letters, 22, 423-436.

Levin SA (1992) The problem of pattern and scale in ecology: The Robert H. MacArthur award lecture. Ecology, 73, 1943-1967.

Levine JM, Bascompte J, Adler PB, Allesina S (2017) Beyond pairwise mechanisms of species coexistence in complex communities. Nature, 546, 56-64.

Levins R (1968) Evolution in Changing Environments: Some Theoretical Explorations. Princeton University Press, Princeton.

Lewontin RC (1969) The meaning of stability. Brookhaven Symposium in Biology, 22, 13-24.

Li A, Cornelius SP, Liu YY, Wang L, Barabási AL (2017) The fundamental advantages of temporal networks. Science, 358, 1042-1046.

Li DZ, Liu KY, Zang RG, Wang XP, Sheng LJ, Zhu ZL, Shi Q, Wang CA (2006) Development of the modern niche theory and its main representative genres. Scientia Silvae Sinicae, 42(8), 88-94. (in Chinese with English abstract) [李德志, 刘科轶, 蔵润国, 王绪平, 盛丽娟, 朱志玲, 石强, 王长 爱 (2006) 现代生态位理论的发展及其主要代表流派. 林业科学, 42(8), 88-94.]

Liu YY, Barabási AL (2016) Control principles of complex systems. Reviews of Modern Physics, 88, 035006.

Liu YY, Slotine JJ, Barabási AL (2011) Controllability of complex networks. Nature, 473, 167-173.

Loreau M, Hector A (2001) Partitioning selection and complementarity in biodiversity experiments. Nature, 412, 72-76.

Loreau M, Naeem S, Inchausti P, Bengtsson J, Grime J, Hector A, Hooper D, Huston M, Raffaelli D, Schmid B, Tilman D, Wardle DA (2001) Biodiversity and ecosystem functioning: Current knowledge and future challenges. Science, 294, 804-808.

Lu PL, Yu Q, He QT (2006) Responses of plant phenology to climatic change. Acta Ecologica Sinica, 26, 923-929. (in Chinese with English abstract) [陆佩玲, 于强, 贺庆棠 (2006) 植物物候对气候变化的响应. 生态学报, 26, 923-929.] 
Margalef R (1968) Perspectives in Ecological Theory. University of Chicago Press, Chicago.

Mariani MS, Ren ZM, Bascompte J, Tessone CJ (2019) Nestedness in complex networks: Observation, emergence, and implications. Physics Reports, 813, 1-90.

May RM (1972) Will a large complex system be stable? Nature, 238, 413-414.

May RM (1975) Stability in ecosystems: Some comments. In: Unifying Concepts in Ecology (eds van Dobben WH, Lowe-McConnell RH), pp. 161-168. Springer, New York.

May RM (2006) Network structure and the biology of populations. Trends in Ecology and Evolution, 21, 394-399.

Meszéna G, Gyllenberg M, Pásztor L, Metz JA (2006) Competitive exclusion and limiting similarity: A unified theory. Theoretical Population Biology, 69, 68-87.

Michalska-Smith MJ, Allesina S (2019) Telling ecological networks apart by their structure: A computational challenge. PLoS Computational Biology, 15, 1-13.

Montoya JM, Pimm SL, Solé RV (2006) Ecological networks and their fragility. Nature, 442, 259-264.

Morgan Ernest S, Brown JH (2001) Homeostasis and compensation: The role of species and resources in ecosystem stability. Ecology, 82, 2118-2132.

Newman MEJ (2010) Networks: An Introduction. Oxford University Press, Oxford.

Niu KC, Liu YN, Shen ZH, He FL, Fang JY (2009) Community assembly: the relative importance of neutral theory and niche theory. Biodiversity Science, 17, 579-593. (in Chinese with English abstract) [牛克昌, 刘怿宁, 沈泽 昊, 何芳良, 方精云 (2009) 群落构建的中性理论和生态 位理论. 生物多样性, 17, 579-593.]

Nordbotten JM, Levin SA, Szathmáry E, Stenseth NC (2018) Ecological and evolutionary dynamics of interconnectedness and modularity. Proceedings of the National Academy of Sciences, USA, 115, 750-755.

Nova N, Deyle ER, Shocket M, MacDonald AJ, Childs M, Rypdal M, Sugihara G, Mordecai EA (2019) Empirical dynamic modeling reveals ecological drivers of dengue dynamics. bioRxiv, doi: 10.1101/2019.12.20.883363.

Odum EP (1969) The strategy of ecosystem development. Science, 164, 262-270.

O’Dwyer JP, Rominger A, Xiao X (2017) Reinterpreting maximum entropy in ecology: A null hypothesis constrained by ecological mechanism. Ecology Letters, 20, 832-841.

Pascual M, Dunne JA (2006) Ecological Networks: Linking Structure to Dynamics in Food Webs. Oxford University Press, Oxford.

Pascual-García A, Bastolla U (2017) Mutualism supports biodiversity when the direct competition is weak. Nature Communications, 8, 14326.

Payrató-Borràs C, Hernández L, Moreno Y (2019) Breaking the spell of nestedness: The entropic origin of nestedness in mutualistic systems. Physical Review X, 9, 31024.
Pearl J (2009) Causality. Cambridge University Press, Cambridge.

Pellissier L, Albouy C, Bascompte J, Farwig N, Graham C, Loreau M, Maglianesi MA, Melián CJ, Pitteloud C, Roslin T, Rohr R, Saavedra S, Thuiller W, Woodward G, Zimmermann NE, Gravel D (2018) Comparing species interaction networks along environmental gradients. Biological Reviews, 93, 785-800.

Petry WK, Kandlikar GS, Kraft NJ, Godoy O, Levine JM (2018) A competition-defence trade-off both promotes and weakens coexistence in an annual plant community. Journal of Ecology, 106, 1806-1818.

Pilosof S, Porter MA, Pascual M, Kéfi S (2017) The multilayer nature of ecological networks. Nature Ecology and Evolution, 1, 1-9.

Pimm SL (1982) Food Webs. Chapman and Hall, London.

Power ME, Tilman D, Estes JA, Menge BA, Bond WJ, Mills LS, Daily G, Castilla JC, Lubchenco J, Paine RT (1996) Challenges in the quest for keystones: Identifying keystone species is difficult-but essential to understanding how loss of species will affect ecosystems. BioScience, 46, 609- 620.

Proulx SR, Promislow DE, Phillips PC (2005) Network thinking in ecology and evolution. Trends in Ecology and Evolution, 20, 345-353.

Ribando JM (2006) Measuring solid angles beyond dimension three. Discrete \& Computational Geometry, 36, 479-487.

Roberts A (1974) The stability of a feasible random ecosystem. Nature, 251, 607-608.

Rohr RP, Saavedra S, Bascompte J (2014) On the structural stability of mutualistic systems. Science, 345, 1253497.

Rohr RP, Saavedra S, Peralta G, Frost CM, Bersier LF, Bascompte J, Tylianakis JM (2016) Persist or produce: A community trade-off tuned by species evenness. The American Naturalist, 188, 411-422.

Rossberg AG (2013) Food Webs and Biodiversity: Foundations, Models, Data. John Wiley \& Sons, London.

Roughgarden J (1975) A simple model for population dynamics in stochastic environments. The American Naturalist, 109, 713-736.

Saavedra S, Cenci S, del Val E, Boege K, Rohr RP (2017a) Reorganization of interaction networks modulates the persistence of species in late successional stages. Journal of Animal Ecology, 86, 1136-1146.

Saavedra S, Rohr RP, Bascompte J, Godoy O, Kraft NJ, Levine JM (2017b) A structural approach for understanding multispecies coexistence. Ecological Monographs, 87, 470-486.

Saavedra S, Rohr RP, Fortuna MA, Selva N, Bascompte J (2016a) Seasonal species interactions minimize the impact of species turnover on the likelihood of community persistence. Ecology, 97, 865-873.

Saavedra S, Rohr RP, Olesen JM, Bascompte J (2016b) Nested species interactions promote feasibility over stability during the assembly of a pollinator community. Ecology and Evo- 
lution, 6, 997-1007.

Saavedra S, Stouffer DB, Uzzi B, Bascompte J (2011) Strong contributors to network persistence are the most vulnerable to extinction. Nature, 478, 233-235.

Sales-Pardo M (2017) The importance of being modular. Science, 357, 128-129.

Scheffers BR, De Meester L, Bridge TC, Hoffmann AA, Pandolfi JM, Corlett RT, Butchart SH, Pearce-Kelly P, Kovacs KM, Dudgeon D, Pacifici M, Rondinini C, Foden WB, Martin TG, Mora C, Bickford D, Watson JEM (2016) The broad footprint of climate change from genes to biomes to people. Science, 354, aaf7671.

Serván CA, Capitán JA, Grilli J, Morrison KE, Allesina S (2018) Coexistence of many species in random ecosystems. Nature Ecology and Evolution, 2, 1237-1242.

Simmons BI, Wauchope HS, Amano T, Dicks LV, Sutherland WJ, Dakos V (2019) Vulnerable species interactions are important for the stability of mutualistic networks. BioRxiv, doi: 10.1101/604868.

Solow AR, Costello C, Beet A (1999) On an early result on stability and complexity. The American Naturalist, 154, 587-588.

Song C, Altermatt F, Pearse I, Saavedra S (2018a) Structural changes within trophic levels are constrained by within-family assembly rules at lower trophic levels. Ecology Letters, 21, 1221-1228.

Song C, Barabás G, Saavedra S (2019a) On the consequences of the interdependence of stabilizing and equalizing mechanisms. The American Naturalist, 194, 627-639.

Song C, Rohr RP, Saavedra S (2017) Why are some plant-pollinator networks more nested than others? Journal of Animal Ecology, 86, 1417-1424.

Song C, Rohr RP, Saavedra S (2018b) A guideline to study the feasibility domain of multi-trophic and changing ecological communities. Journal of Theoretical Biology, 450, 30-36.

Song C, Rohr RP, Saavedra S (2019b) Beware z-scores. Journal of Animal Ecology, 88, 808-809.

Song C, Rohr RP, Vasseur D, Saavedra S (2020a) Disentangling the effects of external perturbations on coexistence and priority effects. Journal of Ecology, 108, 1677-1689.

Song C, Saavedra S (2018a) Structural stability as a consistent predictor of phenological events. Proceedings of the Royal Society B: Biological Sciences, 285, 20180767.

Song C, Saavedra S (2018b) Will a small randomly assembled community be feasible and stable? Ecology, 99, 743-751.

Song C, Saavedra S (2020a) Bridging parametric and nonparametric measures of species interactions unveils new insights of non-equilibrium dynamics. bioRxiv, doi: 10.1101/2020.03.02.973040.

Song C, Saavedra S (2020b) Telling ecological networks apart by their structure: An environment-dependent approach. PLoS Computational Biology, 16, e1007787.
Song C, Von Ahn S, Rohr RP, Saavedra S (2020b) Towards a probabilistic understanding about the context-dependency of species interactions. Trends in Ecology and Evolution, 35, 384-396.

Sprockett D, Fukami T, Relman DA (2018) Role of priority effects in the early-life assembly of the gut microbiota. Nature Reviews Gastroenterology and Hepatology, 15, 197-205.

Staniczenko PP, Kopp JC, Allesina S (2013) The ghost of nestedness in ecological networks. Nature Communications, 4, 1391.

Stouffer DB, Bascompte J (2011) Compartmentalization increases food-web persistence. Proceedings of the National Academy of Sciences, USA, 108, 3648-3652.

Strogatz SH (2014) Nonlinear Dynamics and Chaos: With Applications to Physics, Biology, Chemistry, and Engineering. Westview Press, Boulder.

Sugihara G (1994) Nonlinear forecasting for the classification of natural time series. Philosophical Transactions of the Royal Society of London, Series A: Physical and Engineering Sciences, 348, 477-495.

Sugihara G, May R, Ye H, Hsieh CH, Deyle E, Fogarty M, Munch S (2012) Detecting causality in complex ecosystems. Science, 338, 496-500.

Suweis S, Simini F, Banavar JR, Maritan A (2013) Emergence of structural and dynamical properties of ecological mutualistic networks. Nature, 500, 449-452.

Thébault E, Fontaine C (2010) Stability of ecological communities and the architecture of mutualistic and trophic networks. Science, 329, 853-856.

Thom R (1972) Stabilité Structurelle et Morphogenèse. InterÉditions, Paris. (in French)

Tilman D (1982) Resource Competition and Community Structure. Princeton University Press, Princeton.

Tylianakis JM, Morris RJ (2017) Ecological networks across environmental gradients. Annual Review of Ecology, Evolution, and Systematics, 48, 25-48.

Uricchio LH, Daws SC, Spear ER, Mordecai EA (2019) Priority effects and nonhierarchical competition shape species composition in a complex grassland community. The American Naturalist, 193, 213-226.

Ushio M, Hsieh Ch, Masuda R, Deyle ER, Ye H, Chang CW, Sugihara G, Kondoh M (2018) Fluctuating interaction network and time-varying stability of a natural fish community. Nature, 554, 360-363.

Valverde S, Piñero J, Corominas-Murtra B, Montoya J, Joppa L, Solé R (2018) The architecture of mutualistic networks as an evolutionary spandrel. Nature Ecology and Evolution, 2, 94-99.

Vellend M (2016) The Theory of Ecological Communities. Princeton University Press, Princeton.

Voitalov I, van der Hoorn P, van der Hofstad R, Krioukov D (2018) Scale-free networks well done. arXiv, 1811.02071. 
Wang S (2018) Simplicity from complex interactions. Nature Ecology and Evolution, 2, 1201-1202.

Wang S, Brose U (2018) Biodiversity and ecosystem functioning in food webs: The vertical diversity hypothesis. Ecology Letters, 21, 9-20.

Wang S, Lamy T, Hallett LM, Loreau M (2019) Stability and synchrony across ecological hierarchies in heterogeneous metacommunities: Linking theory to data. Ecography, 42, 1200-1211.

Woodward G, Ebenman B, Emmerson M, Montoya JM, Olesen JM, Valido A, Warren PH (2005) Body size in ecological networks. Trends in Ecology and Evolution, 20, 402-409.

Wootton JT (1994) The nature and consequences of indirect effects in ecological communities. Annual Review of Ecology and Systematics, 25, 443-466.

Xing DL, Hao ZQ (2011) The principle of maximum entropy and its applications in ecology. Biodiversity Science, 19, 295-302. (in Chinese with English abstract) [邢丁亮, 郝占 庆 (2011) 最大熵原理及其在生态学研究中的应用. 生 物多样性, 19, 295-302.]

Yeakel JD, Pires MM, Rudolf L, Dominy NJ, Koch PL, Guimarães PR, Gross T (2014) Collapse of an ecological network in ancient Egypt. Proceedings of the National Academy of Sciences, USA, 111, 14472-14477.
Zhang WP, Sha P, Jia X, Chu CJ, Xiao S, Lin Y, Bai YY, Wang GX (2013) Effects of positive plant interactions on population dynamics and community structures: A review based on individual-based simulation models. Chinese Journal of Plant Ecology, 37, 571-582. (in Chinese with English abstract) [张炜平, 潘莎, 贾昕, 储诚进, 肖酒, 林 玥, 白燕远, 王根轩 (2013) 植物间正相互作用对种群动 态和群落结构的影响: 基于个体模型的研究进展. 植物 生态学报, 37, 571-582.]

Zhou SR, Zhang DY (2006) Neutral theory in community ecology. Journal of Plant Ecology (Chinese Version), 30, 868-877. (in Chinese with English abstract) [周淑荣, 张大 勇 (2006) 群落生态学的中性理论. 植物生态学报, 30, 868-877.]

Zhou SR, Zhang DY (2008) A nearly neutral model of biodiversity. Ecology, 89, 248-258.

Zhu BR, Zhang DY (2011) A process-based theoretical framework for community ecology. Biodiversity Science, 19, 389-399. (in Chinese with English abstract) [朱璧如, 张大勇 (2011) 基于过程的群落生态学理论框架. 生物 多样性, 19, 389-399.]

(责任编委: 王少鹏 责任编辑: 黄祥忠) 